\title{
Role of relevant, irrelevant, and redundant information in simple concept transfer*
}

\author{
LORRAINE A. LOW, JACQUELINE FORTIER, \\ WILLIAM MICKALIDE, DAVID PAGE \\ and \\ DIANE TROUMPALOS \\ Framingham State College, Framingham, Mass. 01701
}

The influence of a number of different instances during acquisition upon transfer of a simple affirmative concept was examined. Instances were defined by the levels of the relevant dimension and the presence or absence of an irrelevant or a relevant redundant variable. Transfer stimuli consisted of new levels of both the relevant and irrelevant variables. The presence of a relevant redundant variable enhanced acquisition, and the presence of the irrelevant variable retarded it. Transfer was enhanced with increase in the levels of the relevant dimension during acquisition. The results were interpreted as indicating that the presence of a greater number of levels of the relevant dimension results in the acquisition of a more general concept.

In a concept identification task, training on many different problems results in better transfer than training on just a few (Callantine \& Warren, 1955; Morrisett \& Hovland, 1959). The contradictory findings by Adams (1954) have been accounted for by the failure of the multiple-problem group in that experiment to acquire the concept. There is general agreement that, with a high level of acquisition, training on multiple problems results in easier transfer to new problems.

In these experiments, relevant attributes were varied between problems. In the present experiment, both relevant and irrelevant attributes independently defined problem variation during acquisition. This experiment is concerned with the effect of relevant, irrelevant, and relevant redundant information on transfer of a simple affirmative concept. It has been found that increasing the number of relevant and irrelevant dimensions retards acquisition (Bulgarella \& Archer, 1962), whereas increasing the amount of relevant redundant information enhances it (Bourne \& Haygood, 1959). It is expected that the increase in number of both the relevant and irrelevant attributes will retard acquisition of a simple affirmative concept but will enhance transfer to new attributes. The role of relevant redundant information is more complex. With one redundant variable, it is expected that $S$ will utilize both variables to define the concept. Transfer to a situation in which either variable defines the concept, but not both, will be detrimental to performance. It is therefore expected that adding a relevant redundant variable will enhance acquisition but retard transfer.

\footnotetext{
*This paper is sponsored by Conan Kornetsky, who takes full editorial responsibility for its contents.
}

\section{METHOD \\ Subjects}

The Ss were 36 college students from the introductory psychology course. Six Ss served in each condition.

\section{Design}

Variations in the stimuli presented during acquisition defined the different treatment conditions. Two levels of the relevant (RL) dimension and three irrelevancy (IR) conditions constituted the 2 by 3 factorial design. Either two or four different shapes constituted the levels of the relevant dimension. In the first irrelevancy condition, color was held constant (IR-C). In the second, four different colors were varied independently of shape (IR-V). In the third condition, color was utilized as the relevant redundant variable ( $R-R)$, although this condition will be included under the more general term irrelevancy. For the R-R condition, a specific color was associated with a particular geometric shape. The identifying number, 2 or 4 , indicates the number of shapes associated with a particular irrelevancy condition. For the specific condition IR-V4, for example, there were four different shapes and four different colors varying independently of each other. The presence or absence of a small square in the center of the geometric figure constituted an added irrelevant dimension for all conditions during acquisition and transfer. Stimuli were identical for all conditions in the transfer task. Two new shapes of the relevant dimension and two new colors of the irrelevant dimension were presented during transfer. The acquisition slides were not presented during transfer.

\section{Procedure}

The stimuli were projected on a screen two at a time. On half of the trials, the two geometric shapes were identical, and on the other half, they were different. The assignment of the same or different shapes to trials was determined randomly, with the restriction that there was an equal number in any block of eight trials.

The Ss were informed of all possible dimensions in which the stimuli varied. They were further instructed that their task was to determine the correct dimension and to write the letter " $S$ " if the stimuli were the same and " $D$ " if they were different, using the correct dimension as a basis for their decision. A response sheet was so constructed as to prevent $S$ from viewing his previous responses. The stimuli were presented for $6 \mathrm{sec}$, followed immediately by feedback in the form of an S or D presented for $4 \mathrm{sec}$. The same general procedure was followed during transfer, except that feedback was omitted. There were 32 acquisition and 32 transfer trials.

\section{RESULTS AND DISCUSSION}

The acquisition score for each $S$ is the total number of correct responses over each block of eight trials. An analysis of variance for these acquisition scores was performed on the data. There were no differences associated with levels of the relevant dimension. Irrelevancy was statistically significant, $F(2,30)=5.56$, $\mathrm{p}<.01$. The combined means for IR-C, R-R, and IR-V were $7.18,7.75$, and 6.64 , respectively. Thus, redundant information enhanced performance and irrelevant 


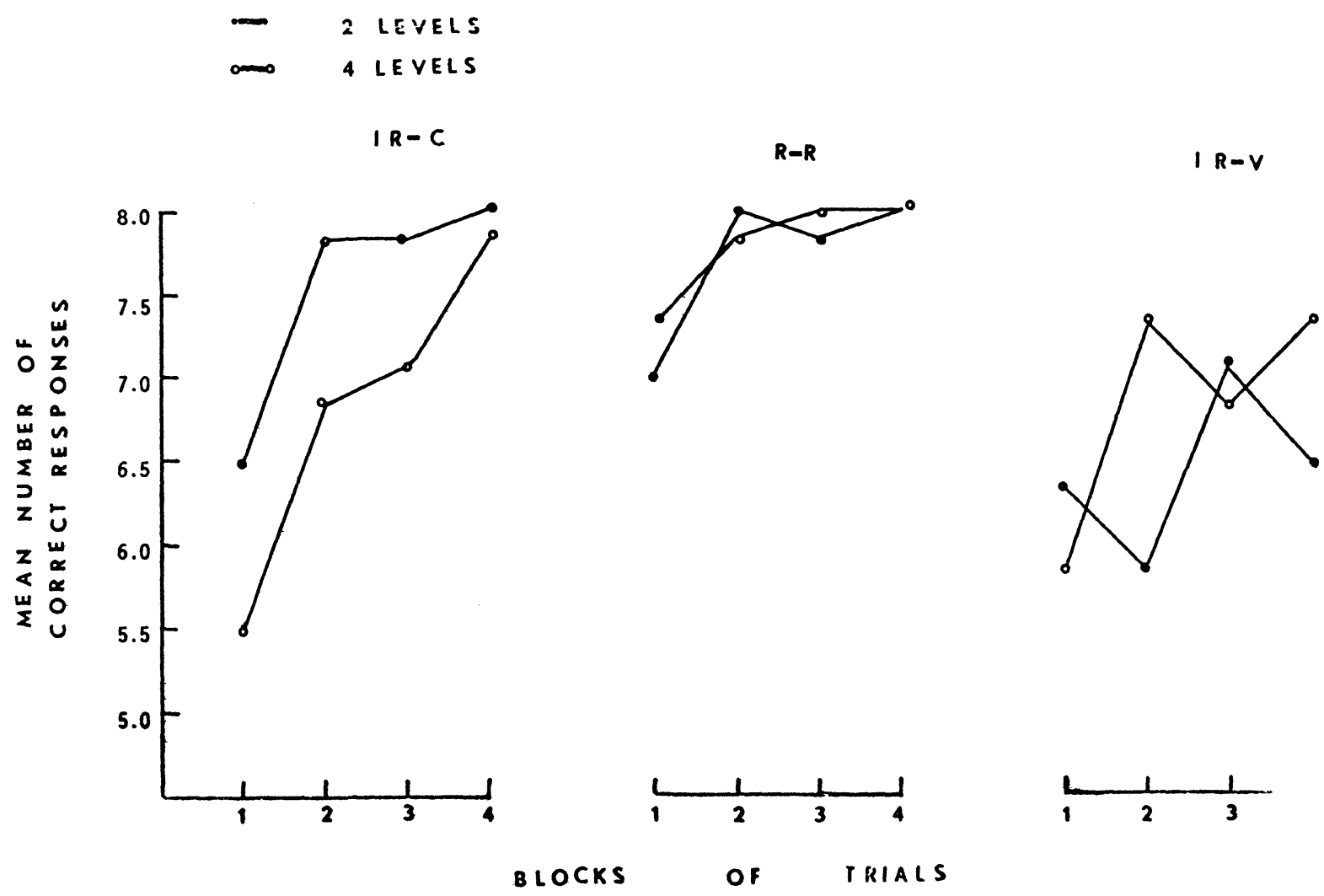

Fig. 1. Mean number of correct responses during acquisition as a function of blocks of eight trials.

information hindered it. The mean number of correct responses during acquisition for each condition is given in Fig. 1.

There was a significant trials effect $[F(3,90)=31.06$, $\mathrm{p}<.01$ ], indicating a general improvement over trials for all conditions. The Trials by RL by IR interaction reached statistical significance $[F(6,90)=2.40$, $\mathrm{p}<.05]$. It is evident from the inspection of Fig. 1 that the IR-C4 group did more poorly than the IR-C2 group but that these differences diminished over trials. Thus, whatever differences were associated with RL tended to diminish over trials when irrelevancy was kept constant. For Group R-R the difference between number of instances (RL) was small from the outset of acquisition. Adding a relevant redundant variable diminished the instances effect. The more variable differences over trials and the ones of greatest magnitude were for groups of IR-V2. It is difficult to account for the poorer and more variable performance of this group.

The transfer measure was obtained by subtracting the number of correct responses over the first 16 transfer trials from the number of correct responses over the last 16 transfer trials. Either color or shape could be used by $\mathrm{S}$ as a basis of classification in the relevant redundant condition. Therefore, the number of correct responses for color and shape was independently computed for
Groups R-R2 and R-R4 during transfer. The largest number of these responses for the first half of transfer was the transfer measure.

In addition to the difference score, the mean number of correct responses over the 32 transfer trials was computed for each S. Since this measure yielded essentially the same results as the difference score, only the results of the latter were reported. The effect of $R L$ was statistically significant $[F(1,30)=7.85, p<.01]$. The mean difference scores for two and four instances were 3.22 and 1.44 , respectively. These differences demonstrate that adding levels to a dimension during acquisition has a positive effect on transfer to new levels of that dimension. These present findings extend previous ones to a simple affirmation concept identification task. The results show that, even in a task involving only one mediating response (shape, in this instance), a greater number of attributes enhance transfer of the concept to new instances. It has been pointed out (Modigliani, 1971) that the mediating response is a necessary, but not a sufficient, condition for formation of the concept. The present findings indicate that the generality of the concept depends not only upon the presence of a mediating response but also upon additional information provided by the number of levels of the relevant dimension. This information, while 
not necessarily utilized in acquisition, influences the generalization of the mediating response to new instances.

There were no differences associated with the irrelevancy variable. The R-R groups, contrary to prediction, displayed no decrement in transfer performance relative to the other groups.

It is possible that the removal of redundancy would have a detrimental effect on transfer with a more difficult concept. The results for the IR-V conditions were in the expected direction only at the four-instance level (IR-V4). The mean difference scores for Groups IR-C4, R-R4, and IR-V4 were 1.83, 2.00, and .50, respectively. It is possible that differences for the two-instance level might have been obscured by the generally poorer performance of Group IR-V2 (see Fig. 1).

\section{REFERENCES}

Adams, J. A. Multiple versus single problem training in human problem solving. Journal of Experimental Psychology, 1954, $48,15-19$

Bourne, L. E., Jr., \& Haygood, R. C. The role of stimulus redundancy in the identification of concepts. Journal of Experimental Psychology, 1959, 58, 232-238.

Bulgarella, R., \& Archer, E. J. Concept identification of auditory stimuli as a function of amount of relevant and irrelevant information. Journal of Experimental Psychology, 1962, 63. 254-257.

Callantine, M. R., \& Warren, J. M. Learning sets in human concept formation. Psychological Reports, 1955, 1, 363-367.

Modigliani, V On the conservation of simple concepts: Generality of the affirmation rule. Journal of Experimental Psychology, 1971, 87, 234-240.

Morrisett, L., \& Hovland, C. I. A comparison of three varieties of training in human problem solving. Journal of Experimental Psychology, 1959, 58, 52-55.

(Received for publication January 15, 1973.) 